\title{
Detection of Human Papillomavirus DNA by the Hybrid Capture Assay
}

\author{
Maria Odete O. Carvalho, Ricardo W. Almeida, \\ Fátima M. S. Leite, Ilza B. Fellows, Mariza H. Teixeira, \\ Ledy H. S. Oliveira and Silvia M. B. Cavalcanti
}

\author{
Department of Microbiology and Parasitology, \\ Biomedical Institute of Universidade Federal \\ Fluminense and Department of Molecular Biology, \\ Laboratórios Sergio Franco, Rio de Janeiro/RJ, Brazil
}

\begin{abstract}
Human Papillomavirus (HPV) infection is the main cause of cervical cancers and cervical intraepithelial neoplasias (CIN) worldwide. Consequently, it would be useful to evaluate HPV testing to screen for cervical cancer. Recently developed, the second-generation Hybrid Capture (HCA II) test is a non-radioactive, relatively rapid, liquid hybridization assay designed to detect 18 HPV types, divided into high and low-risk groups. We evaluated 1055 women for HPV infection with the HCA II test. Five hundred and ten $(48.3 \%)$ of these women had HPV infection; 60 (11.8\%) had low cancer-risk HPV DNA; $269(52.7 \%)$ had high-risk HPV types and $181(35.5 \%)$ had both groups. Hence, 450 women $(88.2 \%)$ in this HPV-infected group had at least one high risk HPV type, and were therefore considered to be at high risk for cancer. Among the group with Papanicolaou (Pap) test results, the overall prevalence of HPV DNA was 58.4\%. Significant differences in HPV infection of the cervix were detected between Pap I (normal smears) and Pap IV (carcinomas) ( $p<0.0001)$. Values of HPV viral load obtained for Pap I and SILs were significantly different, with an upward trend $(p<0.0001)$, suggesting a positive correlation between high viral load values and risk of SIL. Because of the high costs of the HCA II test, its use for routine cervical mass screening cannot be recommended in poor countries. Nevertheless, it is a useful tool when combined with cytology, diagnosing high-risk infections in apparently normal tissues. Use of this technique could help reduce the risk of cancer.
\end{abstract}

Key Words: HPV, SIL, cancer, hybrid capture.

Human Papillomavirus (HPV) infection is the main cause of most cervical cancers and cervical intraepithelial neoplasias (CIN) worldwide [1]. Consequently, it would be useful to evaluate HPV testing for cervical cancer screening. This would require further improvements to and standardization of testing methods. HPV detection has generally been accomplished by hybridization and PCR. But neither research assays nor commercial kits (dot blot or in situ hybridization) have been found adequate for clinical use. An assay for routine clinical use requires reliable

Received on 07 June 2002; revised 22 July 2002.

Address for correspondence: Dr. Silvia Maria Baeta Cavalcanti, Dep. de Microbiologia e Parasitologia, Instituto BiomédicoUFF. Rua Prof. Ernani Melo, 101, $3^{\circ}$ andar (Virologia) 24210130, Niterói, RJ.E-mail: silviabc@ism.com.br.

The Brazilian Journal of Infectious Diseases 2003;7(2):121-125 (C) 2003 by The Brazilian Journal of Infectious Diseases and Contexto Publishing. All rights reserved. and accurate detection of a broad range of pathogenic HPV types that infect the genital tract [2].

Diagnosis of cervical disease, determined by the presence of abnormal cervical epithelial cells, is usually obtained by microscopic examination of Papanicolaou (Pap)-stained smears. This has been the method of choice since the 1950s, proving valuable for mass screening and enabling detection of lesions early enough for effective treatment. The Pap smear has some problems, however. The most important ones are its limited sensitivity for detecting cancer precursors, and the subjective interpretation of results. As a consequence, there are from $20 \%$ to $30 \%$ falsenegatives, and women who have a false-negative diagnosis can develop cervical cancer [3]. Additional methods that could improve the diagnosis of cervical disease have been sought for the past two decades.

Recently developed, the second generation of the Hybrid Capture System HPV DNA detection test from 
Digene Diagnostics (Silver Spring, Md.) is a nonradioactive, relatively rapid, liquid hybridization assay designed to detect $18 \mathrm{HPV}$ types divided into high and low-risk groups. This test has an additional advantage, as it is also designed to provide quantitative estimates of viral load, which may correlate with the grade and the natural history of cervical pathology $[4,5]$.

Given the fact that various clinical laboratories are currently using this method, we decided to use it to make an epidemiological study of HPV infection in cervical lesions, and to correlate the prevalence of HPV with the respective viral load, and with the cytological diagnosis. Such data will permit objective interpretation of this new procedure, and could contribute to cancer prevention.

\section{Materials and Methods}

Study Population and Collection of Specimens. The study population included 1,055 women attended at Laboratórios Sérgio Franco, Rio de Janeiro, from January to December 2000. These women were there for routine exams. The cervical smears were collected by using a cervical cytobrush, and transported in Digene Specimen Transport Medium (Digene Diag, Md).

Cytologic Test. The Papanicolaou test was developed and smears were classified as grade I (PAP I) for normal epithelium, grade II (PAP II) for minor alterations of cervical cells, grade III (PAP III) for low grade squamous intraepithelial lesions (LSIL) and grade IV (PAP IV) for high grade squamous intraepithelial lesions (HSIL), in situ carcinoma and invasive cancer.

HPV Testing. The assay kit detects high-risk types 16 , $18,31,33,35,39,45,51,52,56,58,59$ and 68 . The low-risk group detects the types most commonly associated with condyloma acuminatum: HPV types 6, 11, 42, 43 and 44. Specimens were treated with sodium hydroxide to hydrolyse specimen RNA and to denature the DNA, according to the kit protocol. The liberated single strand DNA was hybridized in solution with an RNA probe mix consisting of the high- or the low-risk HPV types. Each reaction mixture, containing any RNA-DNA hybrids that formed, was transferred to a capture tube coated with antibodies to the hybrids, immobilizing them. Bound RNA-DNA hybrids were then reacted with an alkaline phosphatase-conjugated antibody directed against the hybrids. Unreacted material was removed by washing, and a dioxetanebased chemiluminescent compound, Lumi-Phos 530, was added as a substrate for alkaline phosphatase. The light produced by the ensuing reaction was measured with a Luminometer. Light measurements were expressed as relative light units (RLUs)., Sonicated herring sperm DNA, in Digene transporting medium $(100 \mathrm{mg} / \mathrm{ml})$, was used as a negative control. Triplicate specimens of HPV 16 or HPV 11 DNAs at $1.0 \mathrm{pg} / \mathrm{ml}$ served as the positive controls for high- and low-risk probes, respectively.

All RLU measurements for specimens were divided by the mean RLU of the three corresponding positive controls (PCs) to give a ratio of specimen RLU/PC. A ratio of 1.0 or greater was regarded as positive for HPV DNA, and a ratio of less than 1.0 was regarded as negative.

Since the amount of the light produced by the hybrid capture assay is theoretically proportional to the amount of target HPV DNA, these results can be viewed as quantitative.

Statistical Analysis. The significance of the results was determined by using the $\div^{2}$ test for heterogeneity, with the Yate's continuity correction. All analyses were done using Epi Info 6.02 (CDC).

\section{Results}

A thousand and fifty five female cervical samples were investigated for HPV DNA. The average age of the participants was 31.5 years. Five hundred and ten $(48.3 \%)$ of the women presented HPV infection, as detected by Hybrid Capture. Two hundred thirty three out of the $510(45.7 \%)$ infected women were from 21-30 years old; this age interval had the highest rate of HPV infection (Table 1).

The mean age of the HPV-infected patients was determined (Table 2). There were significant 
differences in the mean age of the patients in the different Pap (severity) groups. The Pap I group (22.1) was significantly younger than the severe Pap IV (HSIL) group (22.1 and 44.3 years, respectively, $\mathrm{p}<0.0001$ ). Among the 510 HPV-positive women, $60(11.8 \%)$ had HPV DNA with a low risk for cancer, 269 (52.7\%) had high risk HPV types and $181(35.5 \%)$ had both types. Four hundred and fifty $(88.2 \%)$ of the women were infected by at least one high risk HPV type.

The HPV DNA prevalence was detemined for each cytological diagnosis group (Pap test): the overall prevalence of HPV DNA in the studied group was $58.4 \%$ (265/454) ranging from 29.6\% (8/27) in Pap I to $84.8 \%$ (28/33) in PAP IV (Table 3). Significant differences were detected in HPV infection of the cervix between Pap I (normal smears) and Pap IV (carcinomas) $(\mathrm{p}<0.0001)$.

The prevalence of the different HPV types was determined (Table 3). Low risk types (Group A) were detected in $3.5 \%$ of Pap II, in $7.3 \%$ of Pap II + HPV and in $8.7 \%$ of Pap III cases (LSIL); while in PAP IV, no low-risk HPV was found alone, indicating a downward trend $(\mathrm{p}<0.0001)$. Group B types, comprising high risk HPV types, were detected in most of the cases, with increasing prevalence according to the severity of the cytological diagnosis; from 10\% (17/169) in Pap II to 85\% (28/33) in Pap IV. These differences were significant ( $p<0.00001)$. The mean values of HPV Viral Load(RLU) were measured by HCA II (Table 4). When we compared values obtained for Pap I and LSIL (from 10.9 to 185.0), we found significant differences for low risk HPV infections ( $p<0.0001)$, suggesting an association between viral load and risk of SIL. The same trend was found for high SILs (from 22.1 to 723.1 in HSIL) ( $<<0.00001)$. Multiple infections (Group A/B) gave the highest RLU values, even in PAP I smears, but the differences were still significant (from 64.2/223.4 in PAPI to 345.1/850.7 in HSIL).

\section{Discussion}

The World Health Organization has indicated that cervical cancer is the second cause of malignant neoplasia and death in women worldwide [1]. In Brazil, cervical cancer is still the most frequent cause of death from cancer and this disease has been reported at increasingly younger ages during the last two decades [6]. Some researchers have questioned the reliability of screening for cervical neoplasia using a Papanicolaou smear alone and have suggested that additional methods be used to improve the accuracy of routine diagnosis of cervical lesions [2].

Cervical cytology suffers from relatively high falsenegative rates (low sensitivity), as well as relatively high false-positive rates (low specificity); these are around $20 \%$ and $15 \%$, respectively [7]. Thus, viral testing to reduce false cytological diagnosis is appealing, given the high frequency of cervical cancer in Brazil [8]. Testing for HPV has been shown to detect SILs missed by false-negative Pap smears. Hence, investigators have suggested combining routine cytology and HPV DNA testing in order to improve disease detection rates. An improvement of $23 \%$ was found (68\% with a Pap test alone versus $89 \%$ with combined tests) [2].

We found the HPV lesion distribution to be agedependent, with a continuous upward trend from Pap I to Pap IV (HSIL), clearly showing a multi-step profile of HPV infection that culminates in cancer establishment. The age interval from 20 to 30 years proved to be the most frequently infectdc, corroborating the association of infection with the beginning of sexual behavior [1].

The hybrid capture assay has been proven to be a reliable, accurate and reproducible HPV test method for routine clinical practice $[9,10,11]$. We found $48.3 \%$ of the women to be infected with HPV, similar to other studies [3] using the same methodology: 29.6\% of Pap I cases were HPV positive (Table 3). Another study [3] found $21 \%$. Nearly $90 \%$ of SILs presented HPV DNA. The hybrid capture assay results were strongly associated with SIL likelihood in concurrently obtained cervical smears. We found $10 \%$ of the SILs to be negative for HCA II and $17.4 \%$ of the cytologic results were unrelated to high-risk HPV types (27 PAP II and 7 PAP I). Some cases of disagreement between DNA testing and cytology were observed, but this may not be due to DNA testing error [12]. The disagreements could be due to either error in the cytological diagnosis or true DNA positivity, with no apparent abnormality. 
Table 1. HPV positivity according to the age of the patients $(n=510)$

\begin{tabular}{lcccccc}
\hline Age intervals & $\mathbf{1 1 - 2 0}$ & $\mathbf{2 1 - 3 0}$ & $\mathbf{3 1 - 4 0}$ & $\mathbf{4 1 - 5 0}$ & $\mathbf{5 1 - 6 0}$ & $\mathbf{6 1 - 7 0}$ \\
\hline Number of patients & 98 & 233 & 112 & 46 & 17 & 4 \\
Percent $(\%)$ & 19.2 & 45.8 & 21.9 & 9.0 & 3.3 & 0.8 \\
\hline
\end{tabular}

Table 2. Mean age of HPV infected women divided into groups according to the cytological diagnosis

\begin{tabular}{lccccc}
\hline Diagnosis & Pap I & Pap II & Pap II + HPV & Pap III(LSIL) & Pap IV (HSIL) \\
\hline Mean age of patients & 22.1 & 28.1 & 27.9 & 34.8 & 49.3 \\
\hline
\end{tabular}

Table 3. Prevalence of HPV groups according to the cytological diagnosis

\begin{tabular}{lccccc}
\hline & & \multicolumn{4}{c}{ Hybrid Capture Assay } \\
\cline { 3 - 6 } Cytology & Number of patients & Group A $^{\mathbf{a}}$ & Group B $^{\mathbf{b}}$ & $\mathbf{A ~ + ~}^{\mathbf{c}}$ & Prevalence (\%) $^{-}$ \\
\hline PAP I & 27 & 1 & 4 & 3 & $30 \%$ \\
PAP II & 169 & 6 & 14 & 13 & $19.5 \%$ \\
PAP + HPV & 110 & 10 & 55 & 29 & $83.6 \%$ \\
PAP III (LSIL) & 115 & - & 17 & 28 & $90.4 \%$ \\
PAP IV (HSIL) & 33 & $\mathbf{2 5}$ & $\mathbf{1 5 6}$ & $\mathbf{8 4}$ & $\mathbf{5 8 . 4 \%}$ \\
\hline Total & $\mathbf{4 5 4}$ & &
\end{tabular}

${ }^{a}$ Low risk HPV types 6,11,42,43,44. ${ }^{\mathrm{b}}$ High risk HPV types $16,18,31,33,35,39,45,51,52,56,58,59,68 .{ }^{\mathrm{c}}$ Low and high risk types in mixed infection.

Table 4. Medium values of Viral load (RLU/PC) measured by Hybrid capture assay, and its relation to the cytological diagnosis and to the HPV group

HPV group by

Cytological diagnosis

\begin{tabular}{lccccc}
\cline { 2 - 5 } hybrid capture & PAP I & PAP II & PAP II + HPV & LSIL & HSIL \\
\hline Group A & 10.9 & 51.9 & 206.3 & 185.0 & - \\
Group B & 22.1 & 85.5 & 263.4 & 367.4 & 723.1 \\
Group A/B & $64.2 / 223.4$ & $694.4 / 891.8$ & $510.3 / 824.0$ & $110.7 / 613.75$ & $345.1 / 850.7$ \\
\hline
\end{tabular}

These cases could be vaginal or cervical lesions that were inapparent or incipient. Negative results in HCA II with alterations in Pap test results were also found. These inconsistent results could be due to low HPV genome copy number, infection by untested types or due to unidentified reasons.

We also found that HSILs were exclusively associated with high risk HPVs (Table 3). No lowrisk virus was found alone in the smears. In agreement with our results, recent reports using the HCA II found no severe dysplasia in women with low-risk HPV [13]. High-risk HPV was present in every case of HSIL, showing a 100\% correlation between high-risk HPV infection and cancer risk.

The quantitative information provided by the hybrid capture test was reliable. We found that viral loads increased with SILs (Table 4). Other investigators have found that a high viral load predicts an increased 
probability of histological/cytological diagnosis of SIL [4] and a higher risk of high-grade SIL [14]. Women who had a high viral load, according to the HCA II test, were found to be at significantly greater risk for SIL and carcinoma in Taiwan. A distinct upward trend of high-risk HPV DNA levels was found to be correlated with the histologic grade of the lesion $(\mathrm{p}=$ 0.001); this was very evident for HSIL, and less so for LSIL [5]. Other studies have also shown that the amount of HPV DNA is a useful predictor of progression to cervical carcinoma, and concluded that the risk of cancer increased correspondingly [15]. But there have been conflicting reports about correlation between viral load and risk of SIL [16]. Thus, the quantitative aspects of the hybrid capture test merit further evaluation.

Because of the high costs the use of HPV test, its use in routine cervical mass screening cannot be recommended in poor countries. Nevertheless, it has been shown to be a useful tool when combined with cytology, diagnosing high-risk infections in apparently normal tissues, which could help reduce the risk of cancer. This new methodology merits further evaluation in order to establish its potential for cancer prevention in risk groups.

\section{References}

1. Muñoz N. Human papillomavirus infection and cancer: the epidemiological evidence. J Clin Virol 2000;19:1-5.

2. Ferenczy A. 1995. Viral testing for genital papillomavirus infection. Int J Gynecol Cancer 1995;5:321-8.

3. Callaghan J., Karim S., Mortlock S., et al. Hybrid capture as a means of detecting human papillomavirus DNA from liquid-based cytology specimens: a preliminary evaluation. Brit J Biomed Sci, 2001;58:184-9.

4. Cox J.T., Lorincz A.T., Schiffman M.H. Human papillomavirus testing by hybrid capture appears to be useful in triaging women with a cytologic diagnosis of ASCUS. Am J Obstet Gynecol 1995; 172:946-54.

5. Sun C.A., Liu J.F., Wu D.M., et al. Viral load of high-risk human papillomavirus in cervical squamous intraepithelial lesions. Int J Gynecol Obstet 2002;76:417.

6. Registro de Patologias Tumorais - Instituto Nacional do Câncer - INCa 1995-2000. Ed INCa, Brazil.

7. Morrison $\mathrm{H}$. Human papillomavirus absence predicts normal cervical histopathologic findings with abnormal papanicolaou smears. J Hum Virol 1993;4:283-7.

8. Cavalcanti S.M.B., Zardo L.G., Passos M.R.L., Oliveira L.H.S. Epidemiological aspects of Human papillomavirus infection and cervical cancer in Brazil. J Infection 2000; $40: 80-7$.

9. Schiffman M.H., Kiviat N.B., Burk R.D., et al. Accuracy and interlaboratory reliability of human papillomavirus DNA testing by hybrid capture. J Clin Microbiol 1995;33:545-50.

10. Terry G., Ho L., Londesborough P., et al. Detection of high-risk HPV types by the hybrid capture 2 test. J Med Virol 2001;65:155-62.

11. Castle P.E., Lorincz A.T., Lohnas I.M., et al. Results of human papillomavirus DNA testing with the hybrid capture II assay are reproducible. J Clin Microbiol 2002;40:1088-90.

12. Koutsky L.A., Holmes K.K., Critlow C.W., et al. A cohort study of the risk of cervical neoplasia grade 2 or 3 in relation to papillomavirus infection. $\mathrm{N}$ Engl $\mathbf{J}$ Med 1992;327:1272-8.

13. Khanna N., Brooks S.E., Chen T.T., et al. Human papillomavirus absence predicts normal cervical histopathologic findings with abnormal papanicolaou smears. J Hum Virol 2001;4:283-7.

14. Cusick J., Terry G., Ho L., et al. Human papillomavirus type 16 DNA in cervical smears as a predictor of highgrade cervical intraepithelial neoplasia. Lancet 1992;339:959-60.

15. Josefsson A.M., Magnusson P.K.E., Ylitalo N., et al. Viral load of human papillomavirus 16 as a determinant for development of cervical carcinoma in situ: a nested case-control study. Lancet 2000;355:2189-93.

16. Yamasaki H., Sasagawa T., Basha W., et al. Hybrid captureII and LCR-E7 for HPV typing in cervical cytologic samples. Int J Cancer 2002;94:222-7. 